\title{
Ellis-van Creveld syndrome, Jeune syndrome, and renal-hepatic-pancreatic dysplasia: separate entities or disease spectrum?
}

\author{
L A Brueton, $M$ J Dillon, $R$ M Winter
}

\begin{abstract}
We describe two children with multiple abnormalities, neither of whom fits neatly into a classical diagnostic category, but who show overlapping features of Ellis-van Creveld syndrome, Jeune syndrome, and renal-hepatic-pancreatic dysplasia. It seems possible that these three entities form part of a disease spectrum rather than being distinct conditions.
\end{abstract}

Two patients are reported with complete situs inversus, short ribs, and similar abnormalities of the pelvic bones. One of them has postaxial polydactyly, cleft lip, and accessory oral frenula and the other has retinitis pigmentosa, dysplastic kidneys, and hepatic dysfunction. They illustrate between them features suggestive of Ellis-van Creveld syndrome, Jeune syndrome, and renal-hepatic-pancreatic dysplasia and their diagnoses raise questions of nosology within this group of syndromes.

\section{Case reports}

CASE 1

The proband, a male infant, is the fifth child of healthy, non-consanguineous Muslim parents. The ages of the mother and father were 29 years and 35 years at the birth of this child. The brothers and sisters are normal. The infant was born by spontaneous vaginal delivery at 39 weeks' gestation after an uneventful pregnancy.

The Kennedy Galton Centre, Clinical Research Centre, Northwick Park Hospital, Watford Road, Harrow, Middlesex HAI 3UJ.

L A Brueton, $\mathrm{R} M$ Winter

Department of Paediatric Nephrology, Institute of Child Health, 30 Guilford Street, London WC1N 1EH. M J Dillon

Correspondence to Dr Brueton.

Received for publication 8 August 1989.

Revised version accepted for publication 30 October 1989.
Birth weight was $3130 \mathrm{~g}$ (10th centile) and head circumference $35.0 \mathrm{~cm}$ ( 50 th centile); the length at birth was not recorded. On initial neonatal examination the baby was noted to have postaxial polydactyly of all four limbs and a small, midline, upper cleft lip. After an initial episode of respiratory distress, he was discharged home at nine days. When reviewed at $2 \frac{1}{2}$ months of age, head circumference was $39 \mathrm{~cm}$ (25th centile), length $57 \mathrm{~cm}$ (10th centile), span $54 \mathrm{~cm}$, and upper segment:lower segment ratio 0.96 (normal).

Physical findings included a midline cleft upper lip, accessory oral frenula, dextrocardia, and postaxial polydactyly as follows: right hand, postaxial post minimus; left hand, supernumerary digit arising from the fifth metacarpal; right foot, postaxial tag; left foot, extra digit plus postaxial scar. Urea and electrolytes, liver function tests, and full blood count were all normal. There was no evidence of hyposplenism on a peripheral blood film. Full cardiac assessment confirmed that there was no defect apart from dextrocardia. Significant findings on radiography included both cardiac (fig l) and visceral situs inversus; postaxial hexadactyly of all four limbs with fusion of the extra metacarpals (fig 2) and metatarsals; short, poorly formed ribs and hook-like, downward

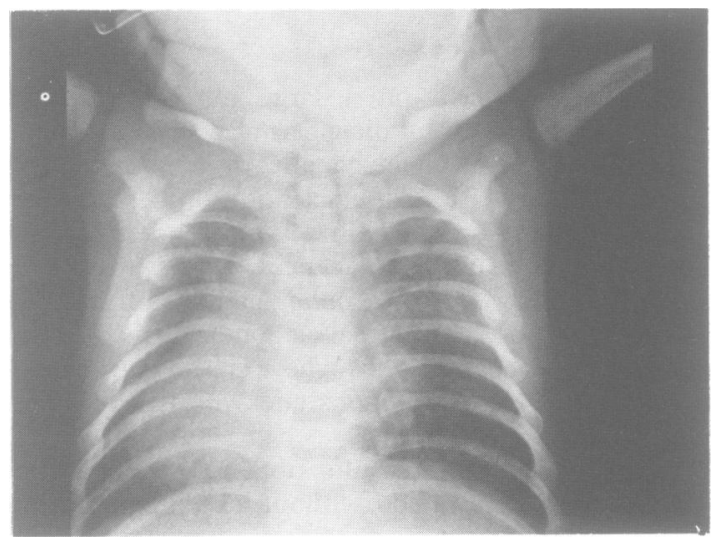

Figure 1 AP supine chest $x$ ray of case 1 showing dextrocardia and poorly formed ribs. 
protrusions from the medial aspects of the acetabula (fig 3). The karyotype was normal at the $400 \mathrm{G}$ band level.

\section{CASE 2}

A Caucasian female infant was delivered at term by emergency caesarian section for breech presentation and failure to progress. Initial respiratory distress

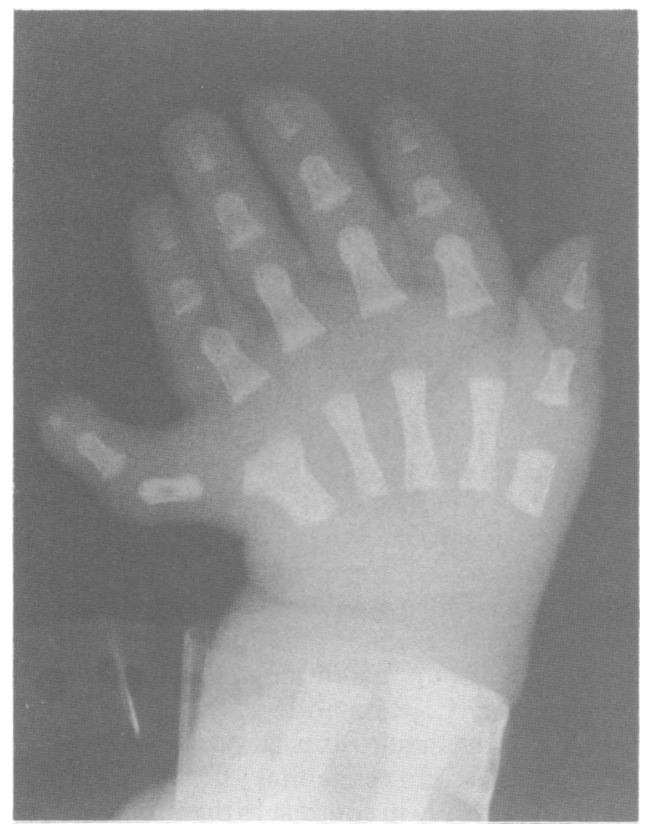

Figure 2 A radiograph of the left hand of case 1 showing postaxial polydactyly with a broad, branched fifth metacarpal.

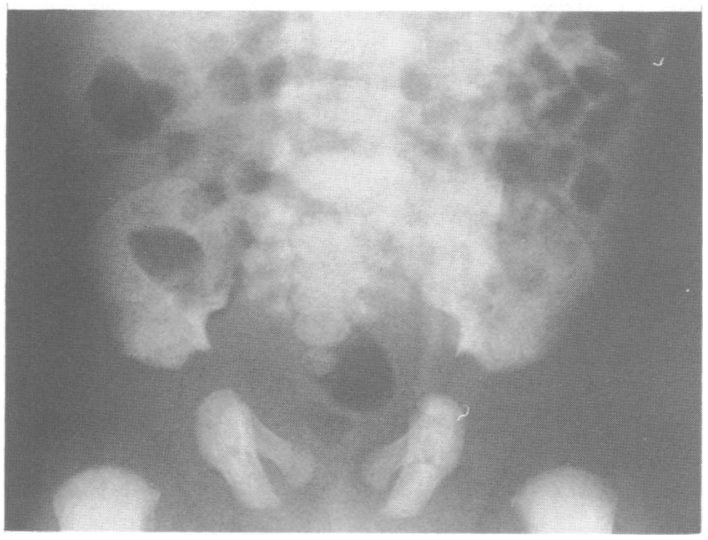

Figure 3 AP supine radiograph of the pelvis of case 1 showing hook-like, downward protrusions from the medial aspects of the acetabula. settled with facial oxygen. On examination she was noted to be hypotonic, mildly dysmorphic with hypertelorism, and to have dextrocardia and a systolic murmur consistent with a VSD. On day 5 the infant developed signs of bowel obstruction with abdominal distension and bile stained vomiting. A low jejunal atresia was found at laparotomy, which was resected, and an anastomosis performed. Postoperatively she became septic and acidotic. Upon investigation she was noted to have a high creatinine of $258 \mu \mathrm{mol} / \mathrm{l}$ and a urea of $7.6 \mathrm{mmol} / \mathrm{l}$. Renal ultrasound showed bilaterally small kidneys with complete loss of cortical/medullary differentiation but no cyst formation. The appearances were consistent with dysplastic kidneys. Echocardiography confirmed the presence of dextrocardia and a VSD and Doppler studies were suggestive of peripheral pulmonary stenosis. Chest $x$ ray showed short ribs and complete situs inversus (fig 4 ), and pelvic $x$ rays showed spur-like, downward projections at the medial and lateral aspects of the acetabular roof (fig 5).

She had a persistent conjugated hyperbilirubinaemia with a peak total bilirubin of $144 \mathrm{mmol} / \mathrm{l}$. An ultrasound of the liver showed it to be slightly enlarged, centrally placed, but of normal echogenicity. Appearances on HIDA scan were suggestive of liver cell dysfunction. Liver biopsy showed a cholangiopathy with hepatic components showing evidence of bile duct damage.

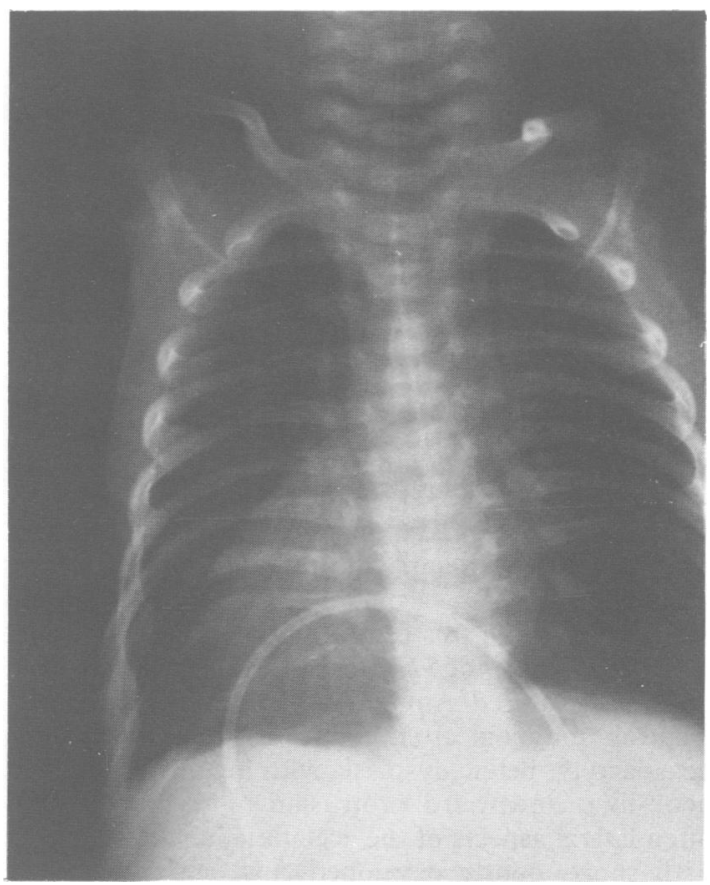

Figure 4 Chest $x$ ray of case 2 showing short ribs and dextrocardia. 


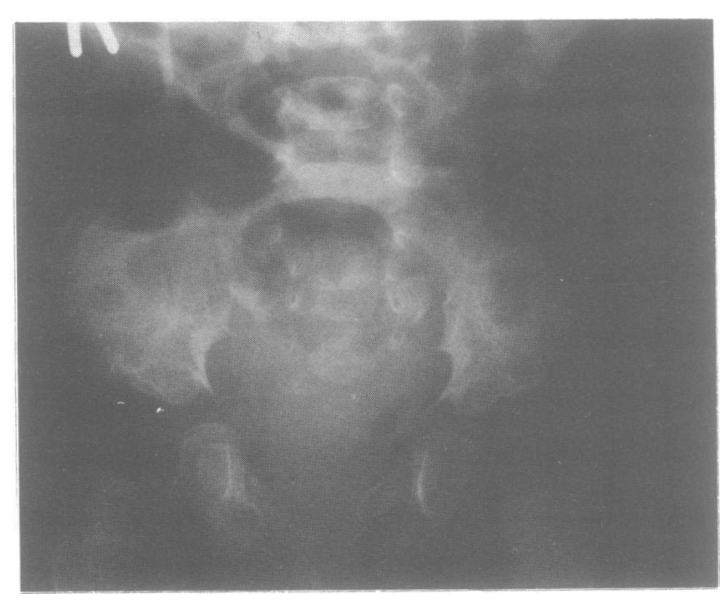

Figure $5 X$ ray of pelvis of case 2 showing spur-like, downward projections at the medial and lateral aspects of the acetabular roof.

Developmental delay was apparent from an early stage and she had roving eye movements and was unable to fix or follow. Ophthalmological assessment indicated the presence of retinitis pigmentosa. Other investigations, including blood and urinary amino and organic acids, $\alpha_{1}$-antitrypsin, sweat test, CT head scan, skull $x$ ray, and TORCH screen, were normal.

After a complicated postoperative course she was. eventually discharged home with continuing problems of failure to thrive and developmental delay with visual impairment. At discharge length and weight were both below the 3rd centile and head circumference between the $3 \mathrm{rd}$ and 10th centiles.

\section{Discussion}

'Chondroectodermal dysplasia', first described by Ellis and van Creveld ${ }^{1}$ in 1940 , is a rare autosomal recessive condition characterised by short stature, polydactyly, dental abnormalities, small nails, and distal shortening of the limbs (Ellis-van Creveld syndrome, EvC). Around 50 to $60 \%$ of affected subjects have congenital heart defects, most commonly atrial septal defect and often a single atrium. The high mortality rate of infants with this syndrome is largely because of these cardiac malformations. Several articles reviewing this condition have been published including a study of 52 cases of EvC occurring within the highly inbred Amish community in the USA. ${ }^{2}$ Typical radiological findings in infancy include hexadactyly, pelvic dysplasia with low iliac wings and hook-like, downward protrusion of the medial and often lateral aspects of the acetabula, a narrow thorax with short, poorly developed ribs, and progressive distal shortening of tubular bones with short, broad middle phalanges and hypoplastic distal phalanges. ${ }^{3}$
Polydactyly, narrow thorax, and an identical pelvic dysplasia also occur in asphyxiating thoracic dystrophy, an autosomal recessive condition described by Jeune et al. ${ }^{4}$ The major diagnostic criterion of Jeune asphyxiating thoracic dystrophy (JATD) is an abnormally small, narrow thorax with short ribs, which often leads to pulmonary hypoplasia and respiratory insufficiency. The features of JATD, narrow thorax, short ribs, short limbed dwarfism, with abnormalities of pelvic bones, polydactyly, and abnormal teeth, bear a striking resemblance to those of EvC. Limb shortening in JATD as in EvC may be minimal or severe. In infants with JATD without polydactyly the combination of changes in the thorax and pelvis is considered diagnostic. However, in infants with polydactyly, one cannot differentiate radiologically between these two syndromes. ${ }^{3}$ The type of polydactyly (commonly postaxial) is the same in the two conditions; the middle phalanges tend to be short and broad and the distal phalanges hypoplastic in both. Polydactyly is an inconsistent feature in JATD and when present usually affects both hands and feet. Polydactyly of the hand is a constant feature of EvC, but polydactyly in the feet is relatively uncommon. Unilateral polydactyly affecting the left hand and right foot has been described in one case of EvC. ${ }^{5}$ Nail dysplasia and upper lip abnormalities (typically partial cleft upper lip connected by multiple frenula to the alveolar ridge), features of EvC, are not seen in JATD. Dental abnormalities have been described in both conditions although are far commoner in EvC. Retinal degeneration resembling Leber congenital amaurosis is an occasional feature of JATD. Associated congenital heart defects are uncommon in JATD in contrast to their high incidence in EvC. Pulmonary insufficiency resulting from the abnormally small thorax is much more frequent in JATD than in EvC and often leads to neonatal death.

Renal and pancreatic insufficiency are complications seen in older patients with JATD and most have portal abnormalities on liver biopsy or at necropsy, although hepatic symptoms are rare. In a report of necropsy findings ${ }^{6}$ in seven neonates with JATD, microscopic abnormalities typified by expansion of the portal areas with bile duct proliferation and periportal fibrosis were seen in the liver in all cases, and in one case cirrhosis was found. Microscopic cystic changes in the kidneys were seen to a varying degree in all. Pancreatic fibrosis was a variable finding. Chronic renal failure is the commonest cause of death in older patients with JATD. ${ }^{7}$ Patients with EvC may also have renal, hepatic, and pancreatic changes at necropsy; however, findings suggestive of renal/hepatic/pancreatic dysfunction during life are very rare.

Similar renal, hepatic, and pancreatic abnormalities occur in the syndrome of renal-hepatic-pancreatic dysplasia, first described by Ivemark et $a^{8}$ in 1959. A 
study by Bernstein $e t a l^{\dagger}$ reported five cases of renalhepatic-pancreatic dysplasia. All five patients were found, on histopathological examination, to have bilateral renal cystic dysplasia, biliary dysgenesis, pancreatic fibrosis, and cyst formation. Although these five cases were all sporadic, there have been previous reports of affected sibs with renal-hepaticpancreatic dysplasia. ${ }^{8}{ }^{10}$ Bernstein $e t a l l^{9}$ emphasised that the patterns of renal, hepatic, and pancreatic involvement described in the study were indistinguishable from those reported in several syndromes including JATD and EvC. They concluded that the diagnosis of renal-hepatic-pancreatic dysplasia should be one of exclusion and that even when all identifiable syndromes are excluded the remaining cases may not constitute a homogeneous group. It is interesting to note that Ivemark $e t a l^{8}$ in their original description of renal-hepatic-pancreatic dysplasia mentioned the presence of bone changes in the younger sib, which at the time were attributed to rickets. The findings of splenic agenesis and cardiac transposition in one case of renal-hepatic-pancreatic dysplasia, ${ }^{10}$ multiple spleens in another, ${ }^{9}$ and complete situs inversus without congenital heart disease in a third ${ }^{9}$ suggest an association between renal-hepatic-pancreatic dysplasia and polyasplenia, ${ }^{11}$ a complex developmental field defect affecting visceral and cardiac situs, with associated spleen and heart defects.

The cases described above are of two children with multiple abnormalities, neither of which fits neatly in a classical diagnostic category, but which seem to fall into the Ellis-van Creveld syndrome/Jeune syndrome/ renal-hepatic-pancreatic dysplasia/polyasplenia spectrum. They have several significant findings in common, including the same $x$ ray changes in the ribs and pelvis and the presence of complete situs inversus. The features of postaxial polydactyly, midline cleft lip, and accessory oral frenula in case 1 are suggestive of EvC, although polydactyly of the feet is uncommon and complete situs inversus very rare; in fact, to our knowledge, it has been reported only once previously. ${ }^{12}$ In case 2 the combination of changes in the thorax and pelvis in the absence of polydactyly, together with retinal abnormalities and evidence of renal and hepatic dysfunction, suggests the diagnosis of JATD. However, complete situs inversus has not to our knowledge been previously associated with JATD, although it has been reported in cases of renalhepatic-pancreatic dysplasia. ${ }^{9}$
From the discussion above it can be seen that associations between renal-hepatic-pancreatic dysplasia and EvC/JATD and between renal-hepatic-pancreatic dysplasia and situs inversus have been previously documented, while similarities between JATD and EvC syndromes are well known. We feel that these two cases of EvC/JATD with situs inversus, one of which also had renal and hepatic abnormalities, draw all of the above conditions together, illustrating between them features suggestive of each but not typical of one in isolation. As the considerable overlap between these conditions becomes apparent it seems possible that these three entities form part of a disease spectrum with a common aetiology, rather than being distinct conditions.

We are grateful to Dr M J Chapple, Consultant Paediatrician, Luton and Dunstable Hospital for referring case 1 and to Mrs S Kingsley for typing the manuscript.

1 Ellis RW, van Creveld S. A syndrome characterised by ectodermal dysplasia, polydactyly, chondrodysplasia and congenital morbus cordis; report of three cases. Arch Dis Child 1940;15: 65-84.

2 McKusick VA, Egeland JA, Eldridge R, Krusen DE. Dwarfism in the Amish: the Ellis-van Creveld syndrome. Bull Fohn Hopkins Hosp 1964;115:306-36.

3 Spranger JW, Langer LO, Wiedemann HR. Bone dysplasia. An atlas of constitutional disorders of skeletal development. Philadelphia: Saunders, 1974:41-50.

4 Jeune M, Beraud C, Carron R. Dystrophie thoracique asphyxiante de caractere familial. Arch Fr Pediatr 1955;12:886-91.

5 Engle MA, Ehlers KH. Ellis-van Creveld syndrome with asymmetric polydactyly and successful surgical correction of common atrium. Birth Defects 1969;V(4):65-7.

6 Turkel SB, Diehl EJ, Richmond JA. Necropsy findings in neonatal asphyxiating thoracic dystrophy. $\mathcal{F}$ Med Genet 1985;22: 112-8.

7 Bernstein J, Brough AJ, McAdams AJ. The renal lesion in syndromes of multiple congenital malformations. Cerebrohepatorenal syndrome, Jeune asphyxiating thoracic dystrophy, tuberous sclerosis, Meckel syndrome. Birth Defects 1974;X(4): $35-43$.

8 Ivemark BI, Oldefelt V, Zetterström R. Familial dysplasia of kidneys, liver and pancreas. A probably genetically determined syndrome. Acta Paediatr Scand 1959;48:1-11.

9 Bernstein J, Chandra M, Creswell J, et al. Renal-hepaticpancreatic dysplasia: a syndrome reconsidered. Am 7 Med Genet 1987;26:391-403.

10 Crawfurd Md'A. Renal dysplasia and asplenia in two sibs. Clin Genet 1978;14:338-44.

11 Opitz JM. Editorial comment on the paper by de la Monte and Hitchins on familial polyasplenia. Am $\mathcal{F}$ Med Genet 1985;21: 175-6.

12 Donlan MA, Murphy JJ, Brakel CA. Ellis-van Creveld syndrome associated with complete situs inversus. Clin Pediatr 1969;8: 366-8. 